\title{
Research on the Library Seat Management System Based on Zigbee
}

\author{
Hui Yan ${ }^{1,}$ a , Haiyan Hu ${ }^{1, b}$, Pengfei Liu ${ }^{1, a}$, Chenghao Ma ${ }^{1, a}$ \\ ${ }^{1}$ Department of Information Engineering,Jilin Business and Technology \\ College,Changchun,China \\ ayanhui7125@126.com, ${ }^{b}$ hhy1979_711@sina.com
}

\begin{abstract}
.
Along with the third wave of information science and technology, the main points are cloud calculating and the internet of things, and more and more people focus on the development and application of internet of things. Seat intelligent management system, which is based on Zigbee, has solved the problems of how to manage large amounts of seats effectively and quickly, and how to get the information of seats. It fully embodies the characteristics of informatization under the era of the internet of things.
\end{abstract}

Keywords: Zigbee,Seat Management System,UP-Atom510 platform

\section{Introduction}

At present, the libraries of each university all need to provide large amount of seats to meet the students learning needs, but there are always different kinds of problems due to the incomplete information, such as it is quite difficult to get one seat during the peak period of study of mid-term and final examinations, or the low rate of seat utilization caused by unreasonable personnel distribution. Meanwhile, it is impossible for the library staff to spend much manpower and material resources counting the seat information, even can not monitor the current seat conditions. Therefore the seat management system should rely on the JSP web server and Zigbee network, and follow the convenient and quick principle, aim to solve the problem of difficulties in library seat management. The core of the system is to embed techniques of UP-Atom510 platform, and also mix with JSP 
web development, Zigbee network development and Android mobile phone software development, so that to provide better management tools for library staff.

\section{The overall design of system}

The main components of system are shown in Figure 1:

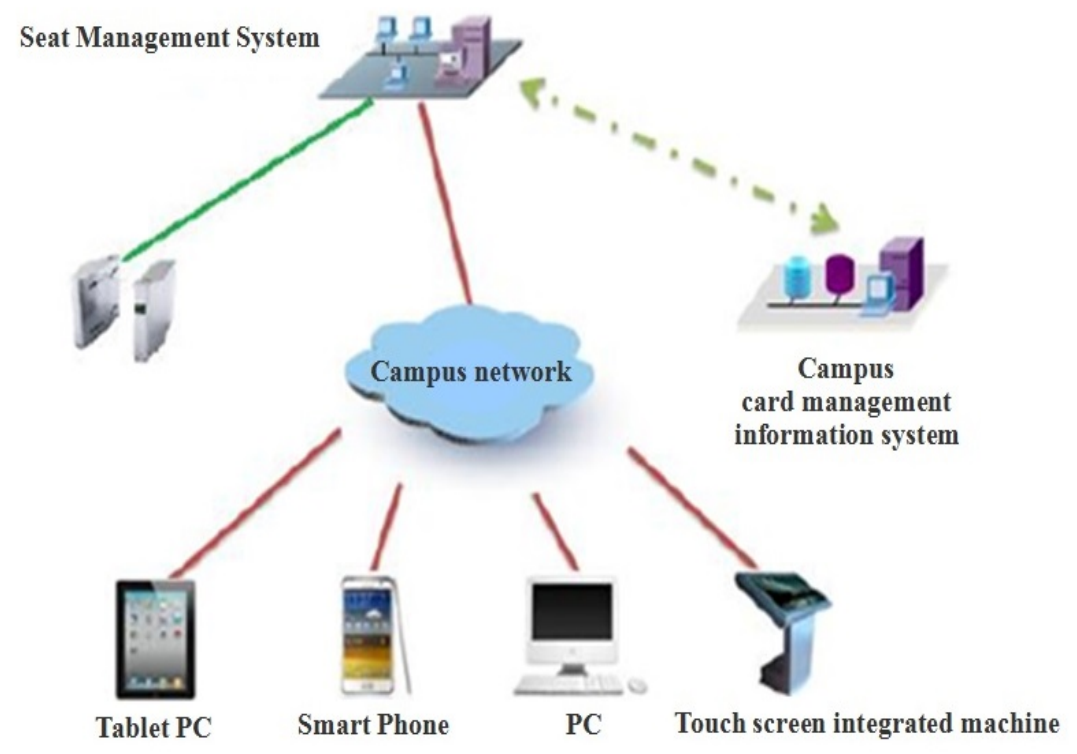

Fig.1 System diagram

\section{Zigbee network}

Zigbee network can realize the function of wireless acquisition on seat status information, the Zigbee network node will be fixed on each seat, and gain the timely situation of seats through sensor, then realize the function of communication between nodes and server by wireless network.

\section{Server}

The server relies on UP-Atom 510 platform to realize the JSP web server side, provides the data interface of Zigbee network and client side, then responses to the 
data requirements from client side, finally realize the function of saving and processing data.

\section{The design of system hardware}

The hardware development platform of seat management system includes: UP-Atom 510 and Zigbee network node.

\section{UP-Atom 510 platform}

The Intel atom platform for teaching and scientific research(model: UP-Atom510) is an embedded experiment platform, which is as a upgraded and substituted product of embedded platform for teaching based on ARM. Meanwhile, it is also can be regarded as the solutions of high-end portable handheld devices, automotive information systems, network interconnection equipment, intelligent video surveillance and industrial control system.

\section{Zigbee network node}

Zigbee network node is composed of human pyroelectric infrared sensor RE200B, micro-controller STC12LE2052AD and Xbee chips.The microcontroller of network node can realize the communication with Xbee module through serial lines, as shown in Figure 2:

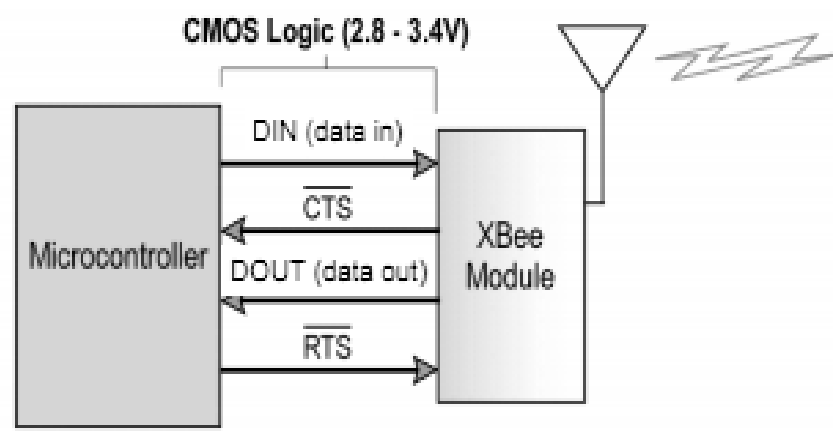

Fig.2 The serial communication model of Zigbee network node The circuit diagram of Zigbee network node is shown in Figure 3: 


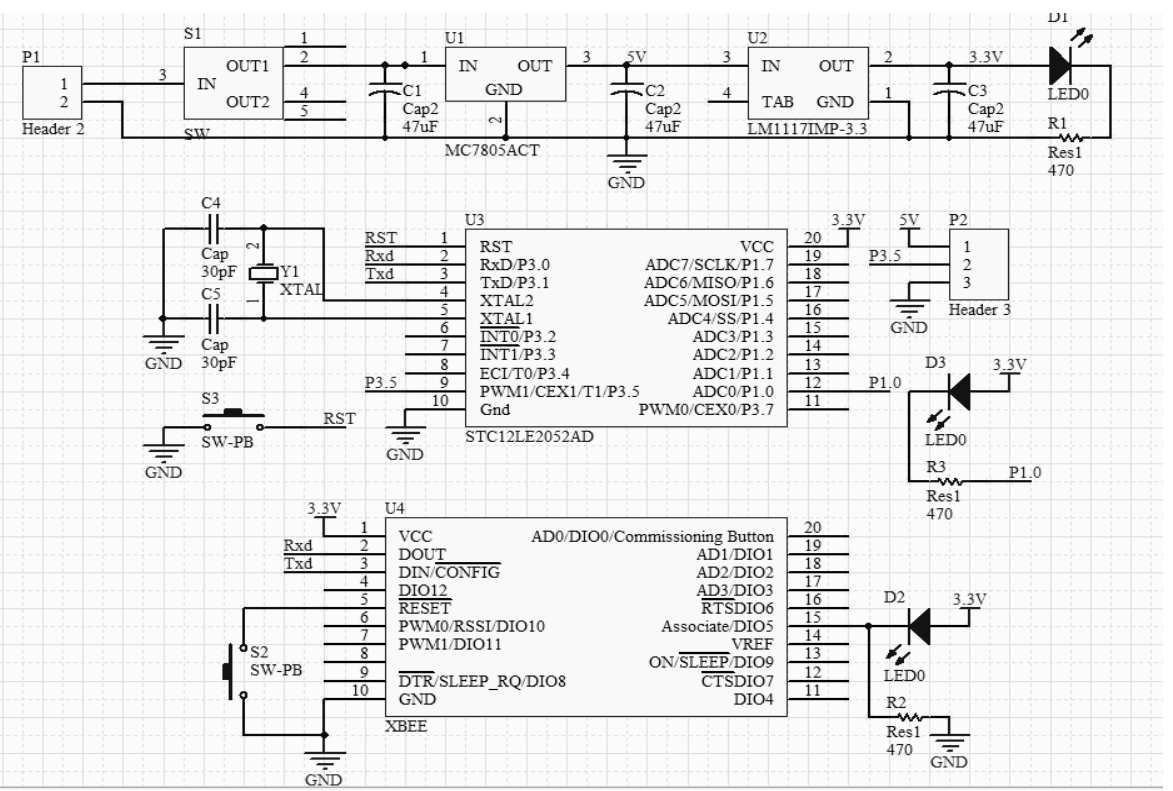

Fig.3 Circuit of Zigbee network node

\section{The design of system software}

\section{The operating system}

The seat management system can support many different kinds of operating system by adopting UP-Atom 510, and in this paper, Ubuntu Server 10.04 is adopted as the operating system.

\section{JSP development platform and database development platform}

The JSP development platform which is adopted by seat management is Tomcat 7.0. Tomcat, a lightweight application server, which is widely used in the small and medium-sized system and the first selection of development and debugging JSP application.

The database development platform of seat management system is SQLite 3. SQLite, mainly for the embedded application, and it occupies less system resources as well as supports the mainstream operating system like Windows, Linux and Unix.

\section{Client side}


The server side is divided into PC clients and Android mobile phone clients, it relies on http protocol to log in the server and then queries and reserves the seat information.

The application process diagram between center nodes and the wireless sensor is as shown in Figure 4.

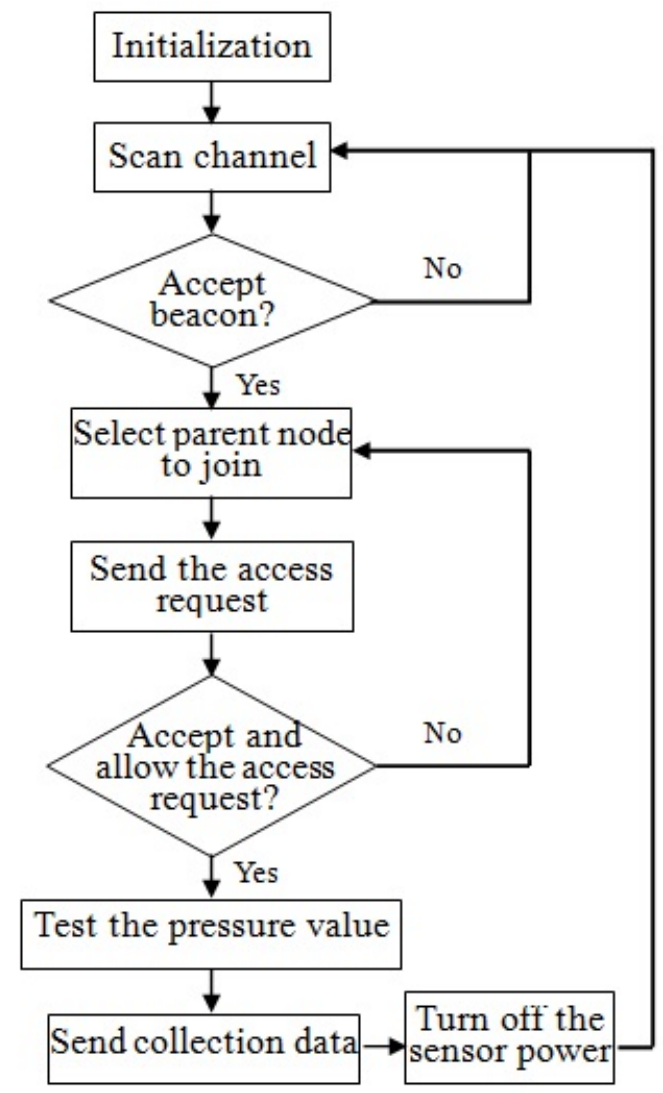

(a) The flow chart of center node 


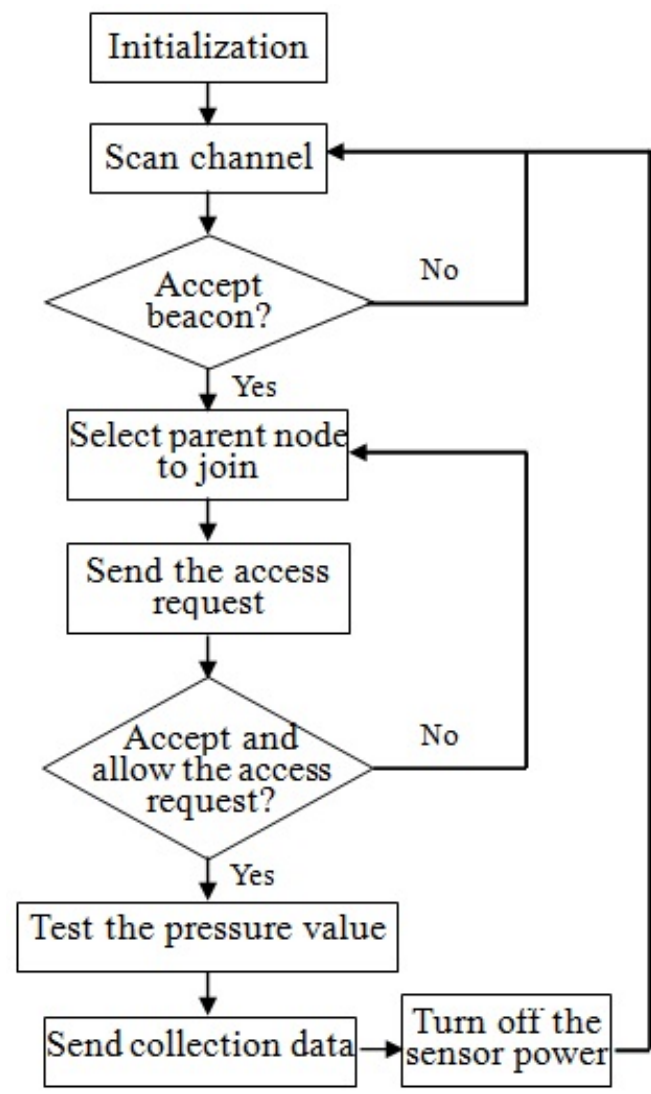

(b) Wireless pressure sensor node

Fig.4 The flow chart of center node and wireless pressure sensor

\section{Conclusion}

In this paper, the seat management system broadens the application range of Zigbee, and gives full play to its performance It realizes the new platform of intelligent seat and systematic management. It can also effectively prevent the phenomenon of taking seats out of order, then alleviate the workload of library staff and the related personal expense to great extent. Meanwhile, it can make more reasonable and orderly use of the resources of libraries.

\section{Acknowledgements}


The authors gratefully acknowledge the fund of this study by Jilin province talent development project in 2013 (20131079) and the Jilin province science and technology department soft science research project (20130420026FG) and the Jilin province education science" The Twelfth Five Year" plan project (GH14496).

\section{References}

[1] Chen Xiangping,Fang Li,Design of data acquisition system based on ZigBee[J],Microcomputer Information,2009,25(10) :99-101.

[2] Sun Dehui,Gong Guanfei,Yang Yang,Design of Wireless Sensor Network System Based on CC2430[J],Modern Electronics Technique,2010,33 (13): 66-68,72.

[3] Zhu Bin,Tang Yong,Tan Yong,Design of data acquisition and transmission system of industrial control network based on ZigBee[J],Control and Instruments in Chemical Industry,2010,37(4)

[4] Cai Wenjing, Qin Huibin, Design of a wireless data acquisition system based on the simplify ZigBee protocol stack[J], Journal of Mechanical \& Electrical Engineering,Vol. 28,No.2. (2011)

[5] Information on http://baike.baidu.com/view/3085090.htm,2012-02-28. 